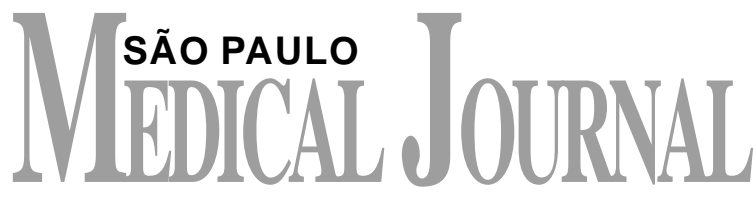

EDITORIAL

Álvaro Nagib Atallah ${ }^{*}$

\section{Essential clinical epidemiology in clinical education}

Some people might consider that to call for physicians to have skills in dealing with the linking of scientific evidence to medical practice in developing countries would be an excessive demand. However, those who are practicing evidence-based medicine nowadays are not ahead of their time but simply up to date. The developed countries are ahead.

In these countries, medical societies are requiring such skills to be in the curriculum for almost all specialities, and particularly in general internal medicine. In the United Kingdom, the Board of the College of Physicians already recommends the teaching of clinical epidemiology and systematic reviews in the residence program and requires these skills in their examinations.

In the United States, the Task Force on the Internal Medicine Residency Curriculum and Graduate Education in Internal Medicine suggests these concepts should be an integral part of the graduation and residency curriculum, in the following words:

\section{"Clinical Epidemiology and Quantitative Clinical Reasoning}

Clinical epidemiology is the study of how clinical questions (such as diagnosis, prognosis, and treatment) are answered by strong scientific research involving populations and groups of patients. Internists must find ways to cope with a rapidly changing evidence-base for medicine, with clinical controversy, and with information overload. They should be able to assess the validity of

* $M D, P h D, M C E$

Chaiman, Department of Internal Medicine, Escola

Paulista de Medicina

Editor, São Paulo Medical Journal. published evidence for themselves. To do so requires understanding the basic clinical research strategies, such as study design, measurement, and analysis, and the meaning of terms used to describe research results in journals. Internists should also be able to judge the credibility of colleagues (authors of review articles, editorials, teachers, and consultants) who synthesize scientific evidence for them. Medical students do not necessarily acquire these abilities in medical school lectures or during teaching rounds; residency programs must teach this material, reinforce it by example, and monitor how well the housestaff use it in clinical care.

Dealing with uncertainty is one of the internist's fundamental skills. Quantitative clinical reasoning, also known as decision analysis, is the best method for using imperfect data to make decisions under conditions of uncertainty. Presented below are clinical epidemiology skills followed by skills of quantitative reasoning.

\section{Competencies for Clinical Epidemiology}

- Understand how bias and chance affect the accuracy of observations on individual patients

- Assess the validity of original research concerning diagnosis, prognosis, treatment, and prevention

- Know the strengths and weaknesses of randomized clinical trials, case-control studies, cohort studies (retrospective, prospective), and meta-analyses

- Demonstrate a practical strategy for judging the validity of colleagues' synthesis of clinical evidence (for example, review articles, continuing medical education courses, or consultant advice)

- Understand the meaning, uses, and limitations of statistical power, $\mathrm{P}$ values and confidence 
intervals, relative risk, attributable risk, and 'number needed to treat'

\section{Competencies for Quantitative Clinical Reasoning}

- Understand how to estimate the pre-test probability of a disease and how to use Bayes' theorem to estimate post-test probability

- Define and use sensitivity, specificity, and likelihood ratios of diagnostic information

- Know and be able to detect potential biases in estimates of sensitivity and specificity

- Understand the value of decision trees and expected value decision making
- Know how to measure patients' preferences

- Understand and utilize sensitivity analysis and cost-effectiveness analysis"

\section{REFERENCE}

Federated Council for Internal Medicine, Task Force on the Internal Medicine Residency Curriculum. Graduate education in internal medicine: a resource guide to curriculum development. Philadelphia: American College of Physicians;1997. ISBN 0-943126-60-6 